\title{
Protective effect of geraniol inhibits inflammatory response, oxidative stress and apoptosis in traumatic injury of the spinal cord through modulation of NF- $\mathrm{NB}$ and p38 MAPK
}

\author{
JIANSHENG WANG, BAISHAN SU, HONGBIN ZHU, CHAO CHEN and GANG ZHAO \\ Department of Orthopedics, The Second Hospital of Tangshan, Tangshan, Hebei 063000, P.R. China
}

Received April 25, 2015; Accepted July 26, 2016

DOI: $10.3892 /$ etm.2016.3850

\begin{abstract}
Geraniol is a type of monoterpenoid with a rose scent and a slightly sweet flavor. It is found in the volatile oil of various plants, and has anti-inflammatory and anti-oxidant effects. The present study aimed to investigate the protective effect of geraniol in inhibiting the inflammatory response, oxidative stress and apoptosis in traumatic spinal cord injury (SCI), as well as to analyze the mechanism underlying its effect. Adult male Sprague-Dawley rats were induced to traumatic SCI through a surgical procedure and were defined as the SCI model group. SCI or normal rats were then administered $250 \mathrm{mg} / \mathrm{kg} /$ day geraniol for 4 weeks. The Basso, Beattie and Bresnahan (BBB) test and the spinal cord water content were used to analyze the effect of geraniol against traumatic SCI in rats. The inflammatory response, oxidative stress, and caspase- 9 and -3 activities were measured using commercial ELISA kits. In addition, the associated mechanism was analyzed, using western blot analysis to determine the protein expression levels of nuclear factor (NF)- $\kappa \mathrm{B}$ and p38 mitogen-activated protein kinase (MAPK). The results of the present study demonstrated that BBB scores were significantly increased and the spinal cord water content was significantly inhibited in SCI rats after 3 weeks of geraniol treatment. Furthermore, the inflammatory response, oxidative stress, and the caspase- 9 and -3 activities were significantly suppressed upon treatment with geraniol. Finally, the mechanism of geraniol against traumatic SCI downregulated the $\mathrm{NF}-\kappa \mathrm{B}$ and p38 MAPK pathways in SCI rats. Therefore, the protective effect of geraniol is suggested to inhibit the inflammatory response, oxidative stress and apoptosis in traumatic $\mathrm{SCI}$ through the modulation of NF- $\mathrm{BB}$ and p38 MAPK.
\end{abstract}

Correspondence to: Mr. Gang Zhao, Department of Orthopedics, The Second Hospital of Tangshan, 21 Jianshen North Road, Tangshan, Hebei 063000, P.R. China

E-mail: ts13717517534@163.com

Key words: geraniol, traumatic injury of the spinal cord, nuclear factor- $\kappa \mathrm{B}, \mathrm{p} 38$ mitogen-activated protein kinase

\section{Introduction}

Traumatic spinal cord injury (SCI) refers to injury resulting from direct or indirect external factors accompanied with motor, sensory and sphincter dysfunction, dystonia and pathological reflex diseases. SCI can be divided into the primary and secondary types (1). Primary SCI is caused by an external force directly or indirectly exerted on the spinal cord (2). Secondary SCI refers to further damages caused by spinal cord compression due to edema, spinal hematoma, compression fracture and fragmentation of the disc, which result from external factors (3). Its clinical symptoms are divided into partial and incomplete; following injury, certain symptoms occur, such as local microvascular spasms, ischemia, increased vascular permeability, small vein rupture and secondary hemorrhagic necrosis (4).

Traumatic SCI usually causes flaccid paralysis, loss of movement, reflexes and sphincter function, impaired microvascular obstruction, hypoxia, spinal cord edema, small congestion in central gray matter necrosis, loss of feeling, and urinary or fecal incontinence (1). A number of symptoms evolve into spastic paralysis after 2-4 weeks, accompanied with an increased muscle tone, tendon hyperreflexia and pathological vertebral tract signs, paraplegia, quadriplegia and flaccid paralysis amongst other symptoms. The movement and deep feeling of an ipsilateral limb below the damaged plane, as well as the contralateral limb pain and the temperature sensation, disappear in traumatic SCI (5). Anterior spinal syndrome is a medical condition with severe compression in the front of the cervical spinal cord, which may occasionally cause central artery occlusion before the spinal cord, as well as quadriplegia. Paraplegia is a more severe condition compared with brachial diplegia, but the sense and feeling of lower limbs and the perineum position remain, and sometimes still preserve a weak sense of feeling (4). The majority of spinal cord syndromes in the area surrounding the central canal occur following cervical hyperextension injury (6). The cervical spinal canal is vulnerable to large volume changes due to cervical hyperextension, and the spinal cord is squeezed by the surrounding wrinkled yellow ligaments, intervertebral discs or bone spurs, which damage the conduction system around the central canal of the spinal cord. This is manifested as quadriplegia below the plane of injury, upper limbs superior to the lower limbs, without poor prognosis (7). 
Aromatherapy in traditional Chinese medicine has been used for the treatment of vaginal candidiasis (8). Geraniol (Fig. 1) is a natural plant source of alcohol that is widely found in citronella, geranium, vanilla and rose oils. It is widely used as a spice ingredient and in cosmetics, fragrances, shampoos, soaps and other non-cosmetic products, including household and other detergents (9). The global use of geraniol is $>1,000$ tons per year. Modern pharmacological studies have revealed that geraniol has a wide range of pharmacological activities, including antibacterial, fungus resistance, insecticidal, antitumor, reverse bacterial resistance and immune regulation properties, while it is also considered to be the pesticide with the minimum environmental risk $(10,11)$.

Therefore, the present study assessed for the first time the protective effects of geraniol in the inhibition of inflammatory response, oxidative stress and apoptosis in traumatic $\mathrm{SCI}$ through modulation of nuclear factor- $\mathrm{\kappa B}(\mathrm{NF}-\kappa \mathrm{B})$ and p38 mitogen-activated protein kinase (MAPK) expression levels.

\section{Materials and methods}

Animals and surgery. Adult male Sprague-Dawley rats (age, 7-8 weeks) were obtained from the Animal Resource Center of the Second Hospital of Tangshan (Tangshan, China). The rats weighted $250 \pm 30 \mathrm{~g}$, and were housed at an ambient temperature of $22 \pm 1^{\circ} \mathrm{C}$ with a 12-h light-dark cycle, and free access to food and water. The present study was performed in accordance with the Guide for the Care and Use of Laboratory Animals of the National Science Council of the Republic of China. The study was approved by the Ethics Committee of the Second Hospital of Tangshan. All experimental procedures attempted to minimize the suffering of the experimental rats. The SCI model was established as described previously (12). Briefly, the experimental rats were anesthetized with intraperitoneal injection of sodium pentobarbital $(50$ mg/kg; Sigma-Aldrich; Merck KGaA, Darmstadt, Germany). Subsequently, a laminectomy was performed at the T10 level to expose the cord beneath it without the dura, the T8 and T9 vertebral peduncles were removed, and SCI was performed during the laminectomy. The sham group rats were subjected to the same procedure without the injury.

Experimental groups and procedures. The experimental rats $(n=40)$ were randomly allocated into four groups of 10 mice each. The groups were as follows: Sham, geraniol-treated, SCI and SCI+geraniol-treated groups. SCI or sham surgery rats received $250 \mathrm{mg} / \mathrm{kg} /$ day geraniol (Sigma-Aldrich) for 4 weeks after surgery, and were defined as the SCI+geraniol-treated or geraniol-treated groups, respectively. In addition, SCI or normal rats were administered an equal volume of normal saline, and were defined as the SCI or sham groups, respectively.

Assessing locomotor recovery. The locomotor recovery was assessed by the Basso, Beattie and Bresnahan (BBB) test subsequent to $\mathrm{SCI}$ operation. A locomotor rating scale of 0 (no observable hind-limb movements) to 21 (normal locomotion) was used (13).
Assessing the spinal cord water content. Spinal cord tissue specimens were collected following geraniol treatment for 4 weeks, and dried for $48 \mathrm{~h}$ at $80^{\circ} \mathrm{C}$ for determining the dry weight. The spinal cord water content was determined as follows: Spinal cord water content $(\%)=($ wet weight - dry weight)/wet weight $x 100 \%$ formula. In addition, SCI or normal rats were administered an equal volume of normal saline, and were defined as the SCI or sham groups, respectively. Rats were sacrificed by decollation under sodium pentobarbital $(50 \mathrm{mg} / \mathrm{kg})$ after 4 weeks of treatment.

Assessing inflammation response. Peripheral blood samples were collected following geraniol treatment for 4 weeks, and centrifuged at $12,000 \times \mathrm{g}$ for $10 \mathrm{~min}$ at $4^{\circ} \mathrm{C}$. The supernatant was collected to assess the activities of serum tumor necrosis factor- $\alpha$ (TNF- $\alpha$; EK0525), interleukin-1 $\beta$ (IL-1 $\beta$; EK0394) and interleukin-6 (IL-6; EK0412), using ELISA kits according to the manufacturer's instructions (Wuhan Boster Biotechnology, Inc., Wuhan, China). The absorbance was measured by a microplate reader at a wavelength of $450 \mathrm{~nm}$.

Assessing oxidative stress. Peripheral blood samples were collected following geraniol treatment for 4 weeks, and centrifuged at $12,000 \mathrm{x} \mathrm{g}$ for $10 \mathrm{~min}$ at $4^{\circ} \mathrm{C}$. The supernatant was collected in order to assess the serum levels of malondialdehyde (MDA; A003-1), superoxide dismutase (SOD; A001-3), glutathione (GSH; A006-2), glutathione peroxidase (GSH-PX; A005) following the manufacturer's instructions (Nanjing Jiancheng Bioengineering Institute, Nanjing, China). The absorbance was measured by a microplate reader (Thermo Fisher Scientific, Inc., Waltham, MA, USA) at a wavelength of $450 \mathrm{~nm}$.

Assessing caspase-9 and caspase-3 concentration in spinal cord tissues. Spinal cord tissue samples were collected following geraniol treatment for 4 weeks, and incubated with $100 \mu \mathrm{l}$ tissue lysis buffer (Beyotime Institute of Biotechnology) for $3 \mathrm{~min}$ on ice. Homogenates were centrifuged at 12,000 x g for $10 \mathrm{~min}$ at $4^{\circ} \mathrm{C}$ and the supernatant was collected to assess the protein concentration using a bicinchoninic acid assay (ww) kit following the manufacturer's instructions (KeyGen Biotech Co., Ltd., Nanjing, China). Equal quantities of protein $(80 \mu \mathrm{g})$ were incubated with Ac-LEHD-pNA (caspase-9; G018) or Ac-DEVD-pNA (caspase-3; G015) at $37^{\circ} \mathrm{C}$ for $2 \mathrm{~h}$ in the dark. The absorbance was measured at a wavelength of $405 \mathrm{~nm}$ using a microplate reader.

Western blot analysis for determination of $N F-\kappa B$ and p38 MAPK expression levels. Spinal cord tissue samples were collected following 4-week geraniol treatment, and incubated with $100 \mu \mathrm{l}$ tissue lysis buffer (Beyotime Institute of Biotechnology) for $30 \mathrm{~min}$ on ice. Homogenates were centrifuged at $12,000 \mathrm{x} \mathrm{g}$ for $10 \mathrm{~min}$ at $4^{\circ} \mathrm{C}$, and the supernatant was collected to assess the protein concentration using a BCA kit following the manufacturer's instructions (KeyGen Biotech Co., Ltd.). Equal protein was separated by $10 \%$ SDS-PAGE and transferred onto $0.22-\mathrm{mm}$ polyvinylidene difluoride (PVDF) membranes (Bio-Rad Laboratories, Inc., Hercules, CA, USA). Each PVDF membrane was blocked with 5\% skim milk powder in Trisbuffered saline plus Tween 20, and incubated with polyclonal 
NF-кB (sc-372; 1:300), polyclonal p-p38 MAPK (sc-7975-R; 1:300) and polyclonal $\beta$-actin primary antibodies (sc-130656; 1:1,000; all Santa Cruz Biotechnology, Inc., Santa Cruz, CA, USA) at $4^{\circ} \mathrm{C}$ for $12 \mathrm{~h}$. Membranes were incubated with the appropriate anti-rabbit horseradish peroxidase-conjugated secondary antibody (C2247; Applygen Technologies, Inc., Beijing, China). The PVDF membrane was then incubated with an enhanced chemiluminescence kit (GE Healthcare Life Sciences, Chalfont, UK). Subsequently, the relative protein expression was determined using an Image Lab 3.0 analysis system (Bio-Rad Laboratories, Inc.).

Statistical analysis. Statistica 17.0 software (StatSoft Holdings, Inc., Taiwan Branch, New Taipei, Taiwan) was used for statistical analysis, using one-way analysis of variance followed by Tukey-Kramer test. The data are expressed as the mean \pm standard error of the mean. $\mathrm{P}<0.05$ was used to indicate a statistically significant difference.

\section{Results}

Protective effect of geraniol on locomotor recovery. The $\mathrm{BBB}$ test was used to analyze locomotor recovery in order to detect whether geraniol had a protective effect in SCI rats. The BBB scores in the SCI group were significantly lower compared with those of the sham or geraniol-treated groups throughout the 4 weeks of the experiment $(\mathrm{P}<0.01$; Fig. 2). After 3 and 4 weeks of geraniol treatment, the BBB scores were significantly increased by pretreatment with geraniol compared with the SCI group $(\mathrm{P}<0.01$; Fig. 2). Thus, geraniol may exert a protective effect on locomotor recovery in TSCI rats.

Protective effect of geraniol on the spinal cord water content. Compared with the sham or geraniol-treated groups, the spinal cord water content was significantly increased in the SCI rats $(\mathrm{P}<0.01$; Fig. 3$)$. This indicated that increase of spinal cord water content induced damage of the spinal cord in TSCI rats. However, after 4 weeks of treatment, treatment with geraniol markedly reduced the spinal cord water content in SCI rats compared with the SCI group $(\mathrm{P}<0.01$; Fig. 3$)$.

Protective effect of geraniol on inflammatory response. Serum TNF- $\alpha$ (Fig. 4A), IL-1 $\beta$ (Fig. 4B) and IL-6 (Fig. 4C) activities were significantly increased following SCI in rats, as compared with the sham or geraniol-treated groups. However, the SCI-induced increase in the serum TNF- $\alpha$, IL-1 $\beta$ and IL- 6 activities was significantly suppressed upon treatment with geraniol in SCI rats $(\mathrm{P}<0.01$; Fig. 4), indicating the protective effect of geraniol on inflammatory response.

Effect of geraniol on the $N F-\kappa B$ protein expression in SCI rats. The protective effect of geraniol against changes in $\mathrm{NF}-\kappa \mathrm{B}$ protein expression was investigated in SCI rats by western blot analysis. The results demonstrated that SCI significantly increased $\mathrm{NF}-\kappa \mathrm{B}$ protein expression compared with that in the sham or geraniol-treated groups $(\mathrm{P}<0.01$; Fig. 5). However, the $\mathrm{NF}-\kappa \mathrm{B}$ protein expression was significantly suppressed by geraniol treatment, when compared with that in the SCI group $(\mathrm{P}<0.01$; Fig. 5$)$.

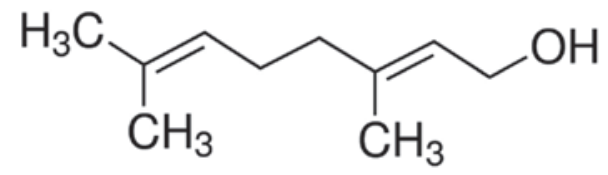

Figure 1. The chemical structure of geraniol.

Protective effect of geraniol against oxidative stress. In order to examine the protective effect of geraniol against oxidative stress following SCI, the serum MDA, SOD, GSH and GSH-PX activities were measured. The results demonstrated a significant increase in the serum MDA activity and a significant reduction in the serum SOD, GSH and GSH-PX activities in SCI rats compared with the sham or geraniol-treated groups (all $\mathrm{P}<0.01$; Fig. 6). As shown in Fig. 6, treatment with geraniol significantly reversed these changes in SCI rats compared with the SCI group $(\mathrm{P}<0.01)$, indicating the protective effect of geraniol against oxidative stress.

Effect of geraniol on the activities of caspase-3 and caspase-9. The caspase-3 and caspase- 9 activities were effectively activated in SCI rats compared with the sham or geraniol-treated groups $(\mathrm{P}<0.01$; Fig. 7). As shown in Fig. 7, treatment with geraniol significantly reduced the caspase- 3 and caspase- 9 activities in SCI rats compared with the SCI group $(\mathrm{P}<0.01)$.

Effect of geraniol on p38 MAPK expression. To examine the protective effect of geraniol on p38 MAPK passage following $\mathrm{SCI}$, the p38 MAPK protein expression was measured by western blotting. When compared with the sham or geraniol-treated groups, a significant increase in p-p38 MAPK protein expression was observed in rats following $\mathrm{SCI}(\mathrm{P}<0.01$; Fig. 8). By contrast, the SCI-induced increase in p-p38 protein expression was significantly inhibited by treatment with geraniol compared with the SCI group $(\mathrm{P}<0.01$; Fig. 8).

\section{Discussion}

Traumatic SCI is a common spinal surgical trauma, characterized by high incidence, high morbidity and low mortality rate, and it greatly affects the quality of life of patients and increases the burden on families $(1,3)$. Recently, the prevalence of traumatic SCI has been increasing on a yearly basis due to the increase in traffic accidents and falls from height amongst other accidents (6). Survey data demonstrated that the annual incidence rate of SCI in Beijing was 68/100,000 individuals (14). The present study investigated the effect of geraniol following traumatic SCI in rats, and the results indicated that geraniol treatment significantly enhanced the BBB scores and reduced the spinal cord water content. Thus, the current study suggested that geraniol may be a potentially new agent for treating traumatic SCI.

Inflammation is a common pathological process of the body, which can occur in various tissues and organs, essentially through the interaction between proinflammatory cytokines and the body (15). In SCI, the immune barrier is damaged and immune cells invade, causing a series of inflammatory reactions, such as endothelial damage, vascular permeability change, edema, inflammatory mediator release, peripheral 


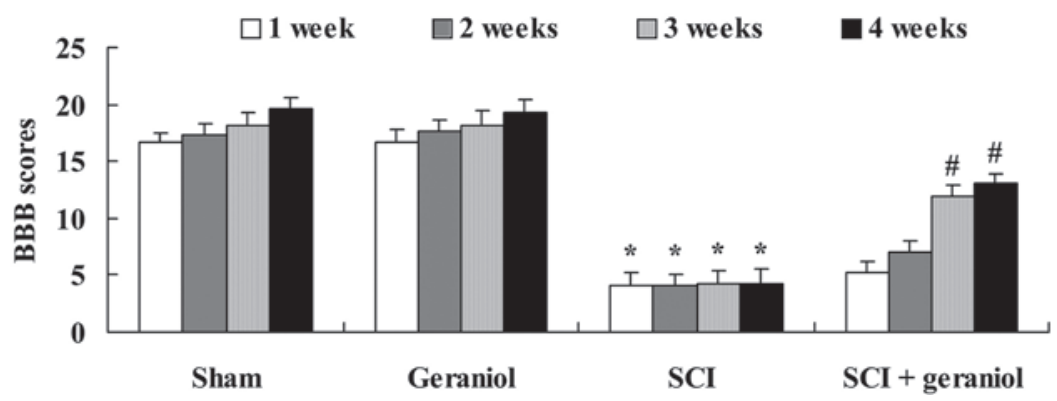

Figure 2. Protective effect of geraniol treatment on locomotor recovery. " $\mathrm{P}<0.01$ vs. sham group; ${ }^{\text {}} \mathrm{P}<0.01$ vs. SCI group. BBB, Basso, Beattie and Bresnahan; SCI, spinal cord injury.

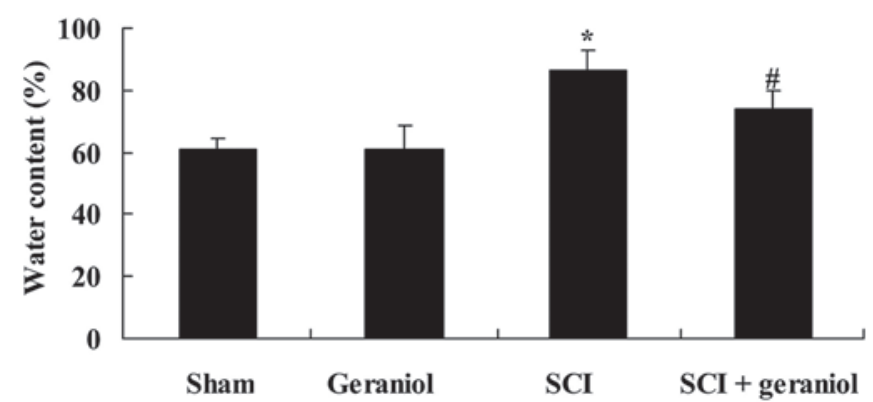

Figure 3. Protective effect of geraniol on the spinal cord water content ${ }^{*} \mathrm{P}<0.05$ vs. sham group; ${ }^{*} \mathrm{P}<0.05$ vs. SCI group. SCI, spinal cord injury.

neutrophil and macrophage chemotaxis, as well as the activation of inherent microglia cells in the spinal cord (16). Immune cells in the spinal cord tissue and peripherally infiltrated neutrophils are able to mediate the damaged immune response and aggravate SCI, but serve an important role in the reconstruction of tissue following SCI (17). Neutrophils are major cells in acute inflammation, which initially enter the site of injury soon after its occurrence, reach a peak at day 1 and begin to decrease after day $3(16,17)$. In the current study, treatment with geraniol significantly suppressed the SCI-induced increase in the serum activities TNF- $\alpha$, IL- $1 \beta$ and IL-6, which are common inflammatory cytokines. Marcuzzi et al (18) reported that geraniol reduces the inflammatory marker levels both in vitro and in vivo. In addition, Chaudhary et al (19) verified that geraniol inhibits murine skin tumorigenesis through inhibition of the oxidative stress response and inflammation. The findings of the present study suggest the anti-inflammation effect of geraniol in treatment for SCI.

$\mathrm{NF}-\mathrm{kB}$ is an important regulator of the expression of inflammatory cytokine genes (20). It is also an important transcription factor, which has a proinflammatory role in the inflammatory response and possesses a variety of biological functions $(21,22)$. $\mathrm{SCI}$ activates NF- $\kappa \mathrm{B}$ in glial, neural and vascular endothelial cells, causing the activation of NF- $\kappa B$. Once activated, NF- $\kappa B$ regulates the expression of a series of immune and inflammation-associated genes at the transcriptional level in the early stage, and also induces a variety of inflammatory cytokines. Drugs used in the treatment of SCI, including methylprednisolone, have demonstrated a significant inhibitory effect on the expression of NF- $\kappa \mathrm{B}$. There is increasing evidence indicating that inhibiting the expression of NF- $\mathrm{\kappa B}$ activity and inflammation is the key for treating SCI (17). However, further research
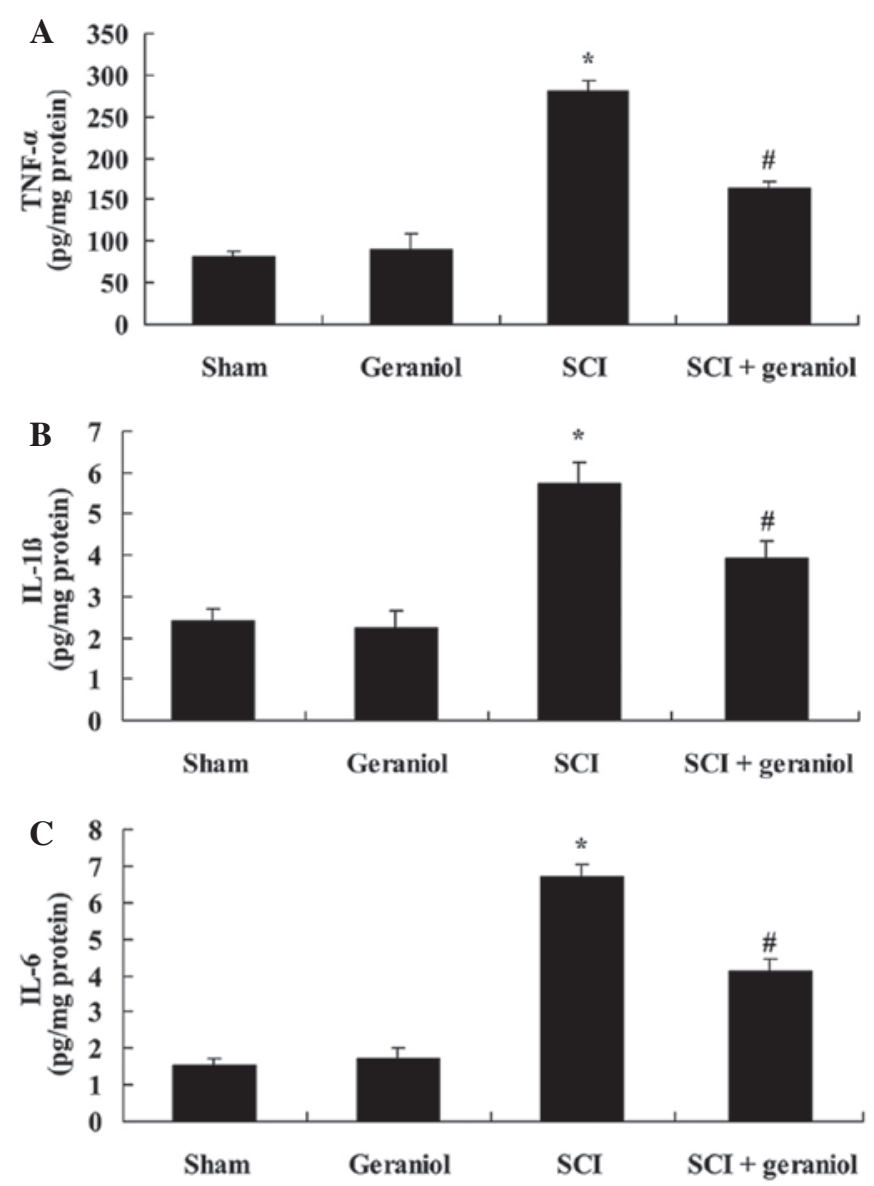

Figure 4. Protective effect of geraniol on inflammatory response, shown by the decrease in SCI-induced serum activities of (A) TNF- $\alpha$, (B) IL- $1 \beta$ and (C) IL-6. " $\mathrm{P}<0.01$ vs. sham group; ${ }^{\#} \mathrm{P}<0.01$ vs. $\mathrm{SCI}$ group. TNF- $\alpha$, tumor necrosis factor- $\alpha$; IL, interleukin; SCI, spinal cord injury.

on NF- $\mathrm{KB}$ revealed that the activation of NF- $\mathrm{KB}$ is not only significant in mediating the spinal cord damage, but it also has extensive functions, including anti-apoptotic protection effects. The present results demonstrated that treatment with geraniol significantly suppressed the NF- $\mathrm{kB}$ protein expression in rats with SCI. Khan et al (23) suggested that geraniol attenuates early tumor promotional changes through $\mathrm{p} 38$ and NF- $\mathrm{KB}$ protein expression. Furthermore, Su et al (24) suggested that geraniol exhibits an anti-inflammatory activity and induces the degradation of NF- $\mathrm{KB}$ p65 in the nucleus. These results indicated that the anti-inflammatory effect of geraniol may function through degradation of NF- $\mathrm{kB}$ p65 in SCI rats. 
A

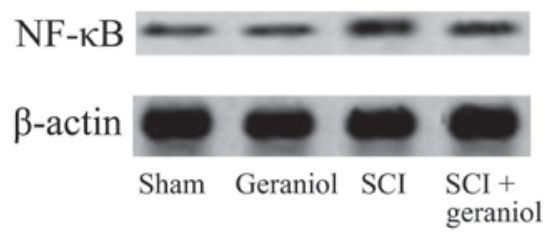

B

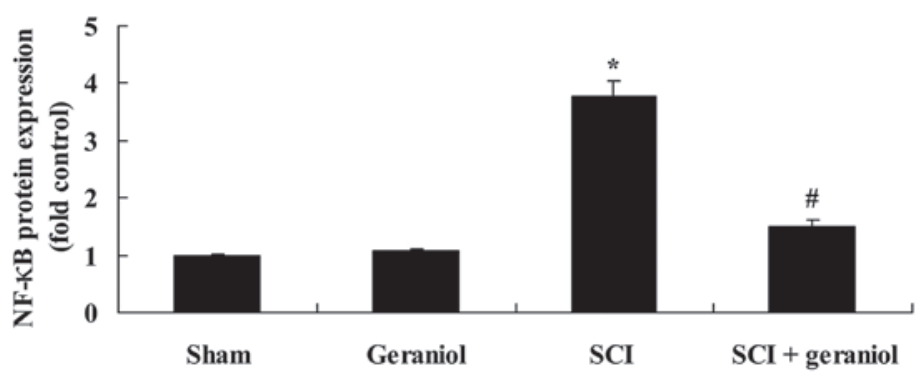

Figure 5. Protective effect of geraniol against SCI-induced increase in NF-kB protein expression, as shown in (A) western blots and (B) quantified results of western blot analysis. ${ }^{*} \mathrm{P}<0.01$ vs. sham group; ${ }^{\#} \mathrm{P}<0.01$ vs. $\mathrm{SCI}$ group. $\mathrm{NF}-\kappa \mathrm{B}$, nuclear factor- $\mathrm{k}$; $\mathrm{SCI}$, spinal cord injury.

A

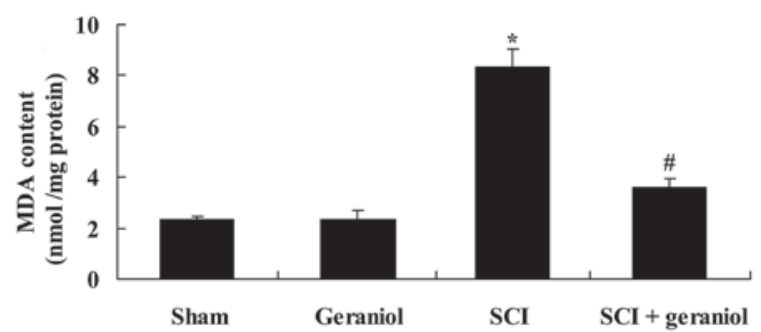

C

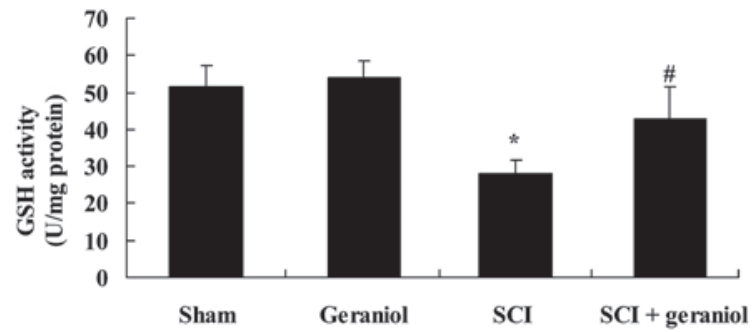

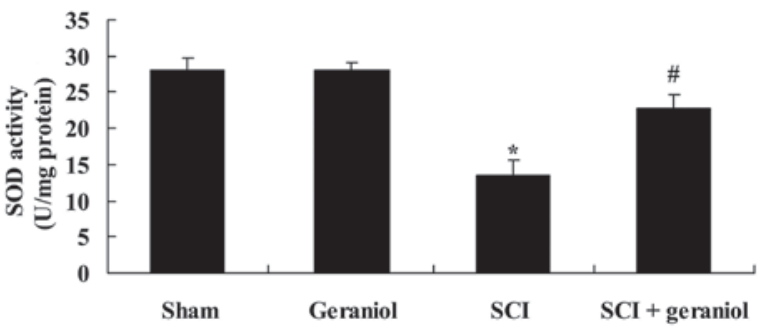

D

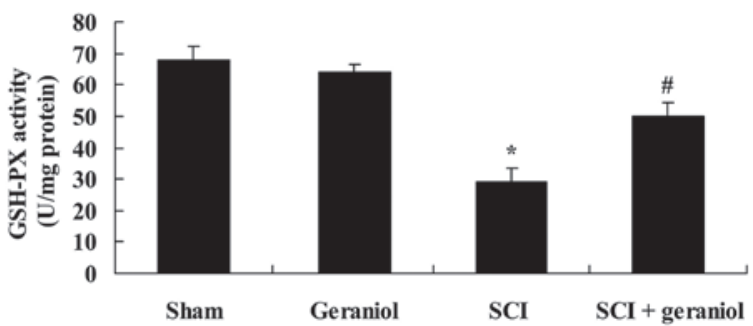

Figure 6. Protective effect of geraniol against oxidative stress. The serum activities of (A) MDA, (B) SOD (C) GSH and (D) GSH-PX were investigated, as indicators of oxidative stress. ${ }^{*} \mathrm{P}<0.01$ vs. sham group; ${ }^{\prime} \mathrm{P}<0.01$ vs. SCI group. MDA, malondialdehyde; SOD, superoxide dismutase; GSH, glutathione; GSH-PX, glutathione-peroxidase; SCI, spinal cord injury.
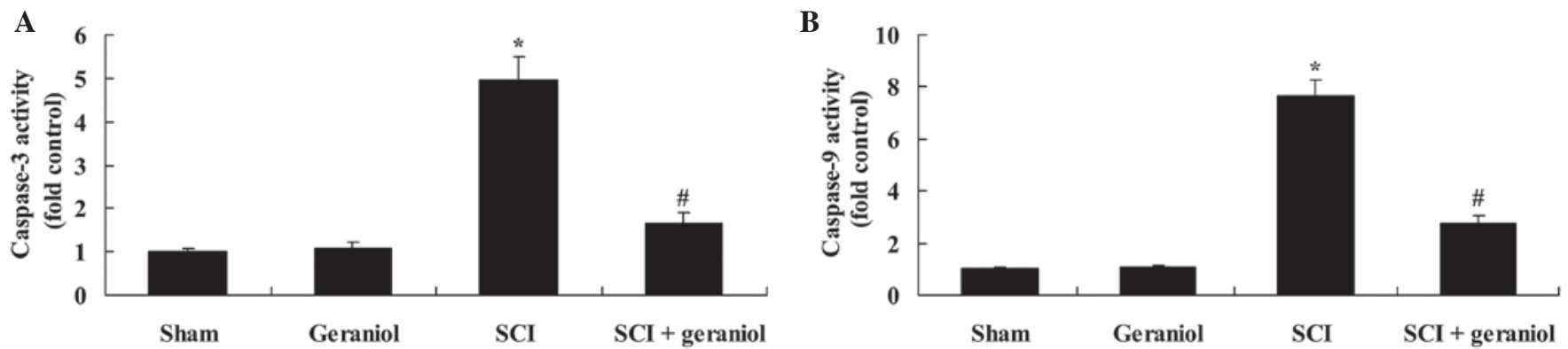

Figure 7. Protective effect of geraniol against changes in (A) caspase- 3 and (B) caspase- 9 activities, induced by SCI treatment. ${ }^{*} \mathrm{P}<0.01$ vs. sham group; ${ }^{\#} \mathrm{P}<0.01$ vs. SCI group. SCI, spinal cord injury.

A

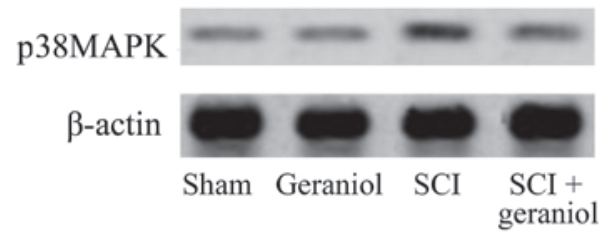

B

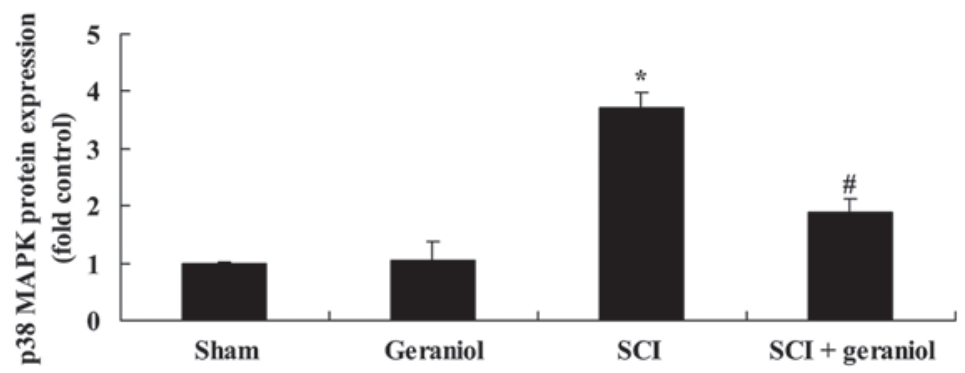

Figure 8. Protective effect of geraniol against changes in p38 MAPK protein expression following SCI, as shown in (A) western blotts and (B) quantified western blot analysis results. " $\mathrm{P}<0.01$ vs. sham group; ${ }^{\mathrm{P}}<0.01$ vs. SCI group. MAPK, mitogen-activated protein kinase; SCI, spinal cord injury. 
The membrane of spinal cord tissue is rich in lipids, and a large number of oxygen-free radicals can be produced and released following damage, which act on the unsaturated fatty acids in the cell membrane, leading to lipid peroxidation and alteration of the membrane permeability (25). In addition, these radicals disintegrate proteasomes, resulting in necrosis and thus causing secondary damage to the spinal cord (25). Intracellular free-radical scavenging mainly depends on two antioxidant systems, including defense enzymatic reactions, such as SOD and GSH-Px. SOD serves a vital role in the body balance between oxidation and antioxidation, which can scavenge free radicals and protect cells from damage (26). In addition, MDA is a decomposition product of lipid peroxidation (27). The body generates oxygen radicals by enzymatic and non-enzymatic systems, which can attack polyunsaturated fatty acids of biofilm, causing lipid peroxidation, and thus forming lipid peroxidation MDA and causing cell damage (27). MDA determination is frequently associated with SOD determination; SOD activity directly reflects the body's ability to scavenge free radicals, while the level of MDA indirectly reflects the severity of the attack on body cells by free radicals (28). The analysis results on SOD and MDA can prove whether the anti-drug oxidation of the body has been increased (26). In the present study, treatment with geraniol significantly reduced the serum MDA activity and increased the serum activities of SOD, GSH and GSH-PX in SCI rats. Hagvall et al (29) indicated that geraniol affects skin sensitization through anti-oxidation. Prasad and Muralidhara (30) also revealed that geraniol may be a promising therapeutic candidate that acts by reducing the levels of oxidative markers in the management of diabetic neuropathy in humans. In the present study, the neuroprotective effect of geraniol against traumatic SCI was shown, which resulted from the anti-oxidative properties of geraniol.

Traumatic SCI is a serious and relatively common injury for which there is no clear and effective approach for treatment (31). Following SCI, necrosis and apoptosis occur in nerve cells and tissues. Apoptosis is a complex pathophysiological process, mainly completed by the protease cascade mediated by caspase family members, in which caspase- 3 serves a key role. Caspase-3 belongs to the cysteine-aspartic protease family that is important in the implementation segment of the apoptosis (31). The present study showed that treatment with geraniol significantly weakened the caspase- 3 and caspase- 9 activities in SCI rats. Similarly, Vinothkumar et al (32) reported that geraniol modulated the apoptosis and inflammation by decreasing the activities of caspase-3 and caspase-9. Thus, the findings of the present study suggested that the anti-apoptotic effect of geraniol has an essential protective role in SCI.

p38 MAPK is known to participate in the signal transduction of neuronal apoptosis, of which the expression strength indicates the survival of nerve cells, and shows an emergency response performance to different extents of cell damage (33). An increase in the expression of p38 MAPK signaling explains the existence of changes in the transduction pathway following SCI in the apoptosis of neurons and glial cells (34). In the present study, geraniol significantly inhibited the increase of p-p38 protein expression of SCI rats. Khan et al (23) suggested that geraniol attenuates early tumor promotional changes through p38 and NF- $\mathrm{B}$. These findings suggest that the modulation of NF- $\kappa \mathrm{B}$ and p38 MAPK may support the neuroprotective effect of geraniol against traumatic SCI.

In conclusion, geraniol treatment exerted a protective role in traumatic SCI by inhibiting the inflammatory response, oxidative stress and apoptosis, thereby promoting functional recovery through downregulation of $\mathrm{NF}-\kappa \mathrm{B}$ and p38 MAPK in SCI rats.

\section{References}

1. Kuo CY, Liou TH, Chang KH, Chi WC, Escorpizo R, Yen CF, Liao HF, Chiou HY, Chiu WT and Tsai JT: Functioning and disability analysis of patients with traumatic brain injury and spinal cord injury by using the world health organization disability assessment schedule 2.0. Int J Environ Res Public Health 12: 4116-4127, 2015.

2. Lin B, Xu Y, Zhang B, He Y, Yan Y and He MC: MEK inhibition reduces glial scar formation and promotes the recovery of sensorimotor function in rats following spinal cord injury. Exp Ther Med 7: 66-72, 2014.

3. Turbes CC: Repair, reconstruction, regeneration and rehabilitation strategies to spinal cord injury. Biomed Sci Instrum 34: 351-356, 1997.

4. Reichenfelser W, Hackl H, Hufgard J, Kastner J, Gstaltner K and Gföhler M: Monitoring of spasticity and functional ability in individuals with incomplete spinal cord injury with a functional electrical stimulation cycling system. J Rehabil Med 44: 444-449, 2012.

5. Emon ST, Irban AG, Bozkurt SU, Akakin D, Konya D and Ozgen S: Effects of parenteral nutritional support with fish-oil emulsion on spinal cord recovery in rats with traumatic spinal cord injury. Turk Neurosurg 21: 197-202, 2011.

6. Vuckovic A, Hasan MA, Fraser M, Conway BA, Nasseroleslami B and Allan DB: Dynamic oscillatory signatures of central neuropathic pain in spinal cord injury. J Pain 15: 645-655, 2014.

7. Tederko P, Krasuski M, Ptyushkin P, Selb M, Pawlak K, Skrzypczyk R and Escorpizo R: Need for a comprehensive epidemiologic study of spinal cord injury in Poland: Findings from a systematic review. Spinal Cord 51: 802-808, 2013.

8. Price S: Specific anosmia to geraniol in mice. Neurosci Lett 4: 49-50, 1977.

9. Lapczynski A, Bhatia SP, Foxenberg RJ, Letizia CS and Api AM: Fragrance material review on geraniol. Food Chem Toxicol 46 (Suppl 11): S160-S170, 2008.

10. Merlini V, Luparia M, Porta A, Zanoni G and Vidari G: Biomimetic cyclization of geraniol derivatives, a useful tool in the total synthesis of bioactive monocyclic terpenoids. Nat Prod Commun 6: 465-476, 2011.

11. Leite MC, de Brito Bezerra AP, de Sousa JP and de Oliveira Lima E: Investigating the antifungal activity and mechanism(s) of geraniol against Candida albicans strains. Med Mycol 53: 275-284, 2015.

12. Hsu JY, McKeon R, Goussev S, Werb Z, Lee JU, Trivedi A and Noble-Haeusslein LJ: Matrix metalloproteinase-2 facilitates wound healing events that promote functional recovery after spinal cord injury. J Neurosci 26: 9841-9850, 2006.

13. Basso DM, Beattie MS, Bresnahan JC, Anderson DK, Faden AI, Gruner JA, Holford TR, Hsu CY, Noble LJ, Nockels R, et al: MASCIS evaluation of open field locomotor scores: Effects of experience and teamwork on reliability. Multicenter Animal Spinal Cord Injury Study. J Neurotrauma 13: 343-359, 1996.

14. Weeks DL, Greer CL, Bray BS, Schwartz CR and White JR Jr: Association of antidepressant medication therapy with inpatient rehabilitation outcomes for stroke, traumatic brain injury, or traumatic spinal cord injury. Arch Phys Med Rehabil 92: 683-695, 2011.

15. Zhang X, Chen C, Ma S, Wang Y, Zhang X and Su X: Inhibition of monocyte chemoattractant peptide-1 decreases secondary spinal cord injury. Mol Med Rep 11: 4262-4266, 2015.

16. Tan J, Zhang F, Liang F, Wang Y, Li Z, Yang J and Liu X: Protective effects of hyperbaric oxygen treatment against spinal cord injury in rats via toll-like receptor $2 /$ nuclear factor- $\kappa \mathrm{B}$ signaling. Int J Clin Exp Pathol 7: 1911-1919, 2014.

17. Yao AH, Jia LY, Zhang YK, Ma QR, Cheng P, Liu L, Ju G and Kuang F: Early blockade of TLRs MyD88-dependent pathway may reduce secondary spinal cord injury in the rats. Evid Based Complement Alternat Med 2012: 591298, 2012. 
18. Marcuzzi A, Crovella S and Pontillo A: Geraniol rescues inflammation in cellular and animal models of mevalonate kinase deficiency. In Vivo 25: 87-92, 2011.

19. Chaudhary SC, Siddiqui MS, Athar M and Alam MS: Geraniol inhibits murine skin tumorigenesis by modulating COX-2 expression, Ras-ERK1/2 signaling pathway and apoptosis. J Appl Toxicol 33: 828-837, 2013.

20. Zuo DC, Choi S, Shahi PK, Kim MY, Park CG, Kim YD, Lee J, Chang IY, So I and Jun JY: Inhibition of pacemaker activity in interstitial cells of Cajal by LPS via NF- $\mathrm{KB}$ and MAP kinase. World J Gastroenterol 19: 1210-1218, 2013.

21. Raha S, Lee HJ, Yumnam S, Hong GE, Saralamma VV, Ha YL, Kim JO, Kim YS, Heo JD, Lee SJ, Eun HK and Kim GS: Vitamin D2 suppresses amyloid-beta 25-35 induced microglial activation in BV2 cells by blocking the NF-kappaB inflammatory signaling pathway. Life Sci: July 28, 2016 (Epub ahead of print).

22. Yarar-Fisher C, Bickel CS, Kelly NA, Stec MJ, Windham ST, McLain AB, Oster RA and Bamman MM: Heightened TWEAK-NF-kappaB signaling and inflammation-associated fibrosis in paralyzed muscles of men with chronic spinal cord injury. Am J Physiol Endocrinol Metab 310: E754-E761, 2016.

23. Khan AQ, Khan R, Qamar W, Lateef A, Rehman MU, Tahir M, Ali F, Hamiza OO, Hasan SK and Sultana S: Geraniol attenuates 12-O-tetradecanoylphorbol-13-acetate (TPA)-induced oxidative stress and inflammation in mouse skin: Possible role of p38 MAP Kinase and NF-кB. Exp Mol Pathol 94: 419-429, 2013.

24. Su YW, Chao SH, Lee MH, Ou TY and Tsai YC: Inhibitory effects of citronellol and geraniol on nitric oxide and prostaglandin $\mathrm{E}_{2}$ production in macrophages. Planta Med 76: 1666-1671, 2010.

25. Xiong Y, Rabchevsky AG and Hall ED: Role of peroxynitrite in secondary oxidative damage after spinal cord injury. J Neurochem 100: 639-649, 2007.

26. Leski ML, Bao F, Wu L, Qian H, Sun D and Liu D: Protein and DNA oxidation in spinal injury: Neurofilaments - an oxidation target. Free Radic Biol Med 30: 613-624, 2001.
27. Knechtle B, Müller G, Willmann F, Eser P and Knecht H: Comparison of fat oxidation in arm cranking in spinal cord-injured people versus ergometry in cyclists. Eur J Appl Physiol 90: 614-619, 2003.

28. Bao F, Chen Y, Dekaban GA and Weaver LC: An anti-CD11d integrin antibody reduces cyclooxygenase-2 expression and protein and DNA oxidation after spinal cord injury in rats. J Neurochem 90: 1194-1204, 2004

29. Hagvall L, Bäcktorp C, Svensson S, Nyman G, Börje A and Karlberg AT: Fragrance compound geraniol forms contact allergens on air exposure. Identification and quantification of oxidation products and effect on skin sensitization. Chem Res Toxicol 20: 807-814, 2007.

30. Prasad SN and Muralidhara: Protective effects of geraniol (a monoterpene) in a diabetic neuropathy rat model: Attenuation of behavioral impairments and biochemical perturbations. J Neurosci Res 92: 1205-1216, 2014.

31. Rodríguez-Barrera R, Fernández-Presas AM, García E, Flores-Romero A, Martiñón S, González-Puertos VY, Mestre H, Flores-Dominguez C, Rodriguez-Mata V, Königsberg M, et al: Immunization with a neural-derived peptide protects the spinal cord from apoptosis after traumatic injury. Biomed Res Int 2013: 827517, 2013

32. Vinothkumar V, Manoharan S, Sindhu G, Nirmal MR and Vetrichelvi V: Geraniol modulates cell proliferation, apoptosis, inflammation, and angiogenesis during 7,12-dimethylbenz[a] anthracene-induced hamster buccal pouch carcinogenesis. Mol Cell Biochem 369: 17-25, 2012.

33. Gong G, Yuan L, Cai L, Ran M, Zhang Y, Gong H, Dai X, Wu W and Dong H: Tetramethylpyrazine suppresses transient oxygen-glucose deprivation-induced connexin 32 expression and cell apoptosis via the ERK1/2 and p38 MAPK pathway in cultured hippocampal neurons. PLoS One 9: e105944, 2014.

34. Kim JH, Choi JS and Lee BH: PI3K/Akt and MAPK pathways evoke activation of FoxO transcription factor to undergo neuronal apoptosis in brain of the silkworm Bombyx mori (Lepidoptera: Bombycidae). Cell Mol Biol (Noisy-le-grand) Suppl 58: OL1780-OL1785, 2012. 